Article

\title{
Hydrodynamics Interactions of Metachronal Waves on Particulate-Liquid Motion through a Ciliated Annulus: Application of Bio-Engineering in Blood Clotting and Endoscopy
}

\author{
Muhammad Mubashir Bhatti ${ }^{1}\left(\right.$ , Asmaa F. Elelamy ${ }^{2}$, Sadiq M. Sait ${ }^{3}$ and Rahmat Ellahi ${ }^{4,5,6, *} *(\mathbb{D}$ \\ 1 College of Mathematics and Systems Science, Shandong University of Science and Technology, \\ Qingdao 266590, Shandong, China; mmbhatti@sdust.edu.cn \\ 2 Department of Mathematics, Faculty of Education, Ain Shams University, El Makrizy Street, Roxy, \\ Heliopolis, 11566 Cairo, Egypt; asmaafathe@edu.asu.edu.eg \\ 3 Center for Communications and IT Research, Research Institute, King Fahd University of Petroleum \& \\ Minerals, Dhahran 31261, Saudi Arabia; sadiq@kfupm.edu.sa \\ 4 Department of Mathematics \& Statistics, FBAS, IIUI, Islamabad 44000, Pakistan \\ 5 Fulbright Fellow Department of Mechanical Engineering, University of California, Riverside, CA 92521, USA \\ 6 Center for Modeling \& Computer Simulation, Research Institute, King Fahd University of Petroleum and \\ Minerals, Dhahran 31261, Saudi Arabia \\ * Correspondence: rellahi@alumni.ucr.edu
}

Received: 17 February 2020; Accepted: 26 March 2020; Published: 3 April 2020

\begin{abstract}
This study deals with the mass transport phenomena on the particle-fluid motion through an annulus. The non-Newtonian fluid propagates through a ciliated annulus in the presence of two phenomenon, namely (i) endoscopy, and (ii) blood clot. The outer tube is ciliated. To examine the flow behavior we consider the bi-viscosity fluid model. The mathematical modeling has been formulated for small Reynolds number to examine the inertia free flow. The purpose of this assumption is that wavelength-to-diameter is maximal, and the pressure could be considerably uniform throughout the entire cross-section. The resulting equations are analytically solved, and exact solutions are given for particle- and fluid-phase profiles. Computational software Mathematica has been used to evaluate both the closed-form and numerical results. The graphical behavior across each parameter has been discussed in detail and presented with graphs. The trapping mechanism is also shown across each parameter. It is noticed clearly that particle volume fraction and the blood clot reveal converse behavior on fluid velocity; however, the velocity of the fluid reduced significantly when the fluid behaves as a Newtonian fluid. Schmidt and Soret numbers enhance the concentration mechanism. Furthermore, more pressure is required to pass the fluid when the blood clot appears.
\end{abstract}

Keywords: cilia motion; blood clot; endoscopy; mass transport; particle-fluid

\section{Introduction}

Flagella and cilia are two distinct names, but are used interchangeably for similar structure of eukaryotic cells. In animals, cilia, which are hair-like appendages, are prominent in the digestive system, respiratory system, reproductive tracts of human beings, and the nervous systems. The movement of cilia plays an essential part in physiological systems, i.e., circulation, respiration, locomotion, alimentation, spermatic fluid propagation, reproduction, etc. It is well-known that ciliary and flagellar movements consist of active sliding, similar to the peristaltic flow of fluid in smooth muscles, whereas flagellar is more complicated. Cilia can be split into two categories, i.e., non-motile and motile. When cilia and flagella are close to each other, they manifest a propagation of 
waves on a large scale known as Metachronal waves. Beating cilia produce metachronal waves over the surface in large numbers, on the ciliated surface of protozoa, and the adjoining activity of cilia coordinates via the hydrodynamics interactions. It is worth mentioning here that metachronal waves are self-organized. Cilia produce bending waves to derive single cells through a medium or to push the fluid over the surface of a fixed cell. The standard form of the ciliary model, motivated by the cilium structure or axoneme, is known as sliding filament model. As shown in Figure 1, the axoneme structure has nine microtubule doublets around the outside, and two are located in the center. Most of the cilia beat at approximately $10-40 \mathrm{~Hz}$, but the form of beating varies. Their length starts from $2 \mu \mathrm{m}$ to several millimeters, and their diameters are approximatley $0.2 \mu \mathrm{m}$. As a result, a low Reynolds number $(\mathrm{Re} \rightarrow 0)$ approximation can be applied.

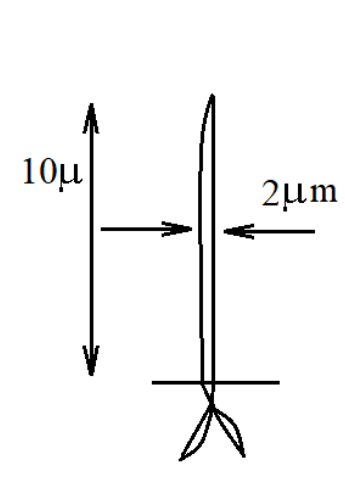

(a)

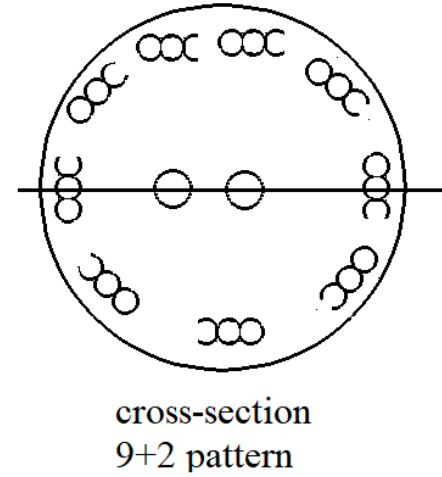

(b)

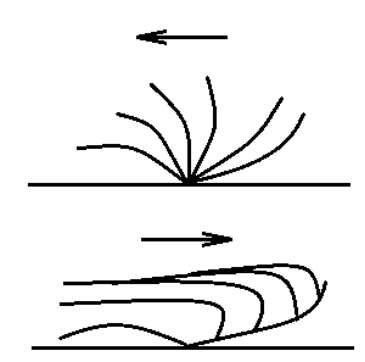

Motion of Cillium Effective recovery stroke

(c)

Figure 1. Strutcure and size of cilia [1] (a) typical dimension, (b) cross-section, (c) cilia stroking.

Nadeem et al. [2] considered the Carreau fluid model to examine the cilia motion through a symmetric channel using the perturbation method. Nadeem and Sadaf [3] discussed the cilia motion of viscous nanofluid through the curvy compliant channel. They used a homotopy analysis method to examine the closed-form solution against the temperature and velocity profile. Maiti and Pandey [4] presented a theoretical study on the nonlinear cilia motion using the Power-law fluid model. Abo-Elkhair et al. [5] used the Adomian decomposition scheme to simluate the cilia motion of magneto-fluid through a ciliated channel. Bhatti et al. [6] discussed the impact of the magnetic field on a ciliated channel using the particle-fluid mechanism. Ashraf et al. [7] examined the peristaltic cilia motion through a human fallopian tube using a Newtonian fluid model. And finally, Ramesh et al. [8] used the behavior of magnetized couple stress fluid model moving through a ciliated channel

Particles in fluid appear in multifarious applications, including biology, geology, chemical engineering, and fluid mechanics [9] to name a few. Several industrial processes include fluidized catalyst beds, pneumatic propagation, and sedimentation. Further, in the biological systems, it involves the flow of blood in the cardiovascular system. The collisions among the particles and the fluids may influence the rheological and the viscosity behavior of the suspension. Particle-wall and particle-particle interactions produce the migration of particles, which causes the anisotropic particle micro-structures and clusters [10,11]. At the mesoscopic scale, a well-known example of the particle-fluid interaction is the movement of the red blood cells (RBC). The flow behavior of the RBC plays a pivotal role in the different pathological and physiological mechanisms. For instance, the rotation and random transverse propagation of RBC in a shear flow plays an essential role in thrombogenesis [12]. These types of movements are firmly associated with the interaction of $\mathrm{RBC}$ to $\mathrm{RBC}$ and fluid (i.e., plasma) to RBC since one $\mathrm{RBC}$ is obstructed by another coming towards it from below or above. RBC is the essential determinant of the blood characteristics in micro-circulation due to their large volume fraction in blood and their aggregability. Mekheimer and Abd Elmaboud [13] investigated the peristaltic motion of fluid having solid particles through different forms of annulus and showed the exact solutions. Mekheimer and Mohamed [14] presented an application of 
a clot blood model using particle-fluid flow through an annulus. Further, they considered the pulsatile flow and obtained analytical solutions. Bhatti et al. [15] discussed the behavior of slip effects using a non-Newtonian fluid model that contains spherical particles. Bhatti and Zeeshan [16] explained the blood flow through an annulus filled with particles and fluid in the presence of a variable magnetic field. Some critical analysis of multiphase simulations are given in the following references [17-21].

Mass transfer with heat on Particle-fluid through fixed and fluidized beds play an essential role and provide necessary information for the development and design of numerous mass and heat transfer operation and chemical reactors including a system of particle and fluid. Gireesha et al. [22] investigated the particle-fluid suspension mechanism through a non-isothermal stretching plate in the presence of a magnetic field and radiative heat flux. They applied a numerical method to obtain the solutions. Bhatti et al. [23] presented a mathematical model of particle-fluid motion induced by a peristaltic wave with thermal radiation and electromagnetohydrodynamics effects. Kumar et al. [24] considered a particle-fluid motion with a nonlinear Williamson fluid model towards a stretching sheet with heat transfer effects. Bhatti et al. [25] explored the particle-fluid motion with heat and mass transfer using Sisko fluid model through a Darcy-Brinkman-Forchheimer porous medium. Some relevant studies on particle-fluid with mass and heat transfer are given in the references [26-28].

The main goal of the present study is to examine the mass transport on the particle-fluid suspension through a ciliated annulus with endoscopy and blood clot effects. Endoscopy plays an essential role in exploring the problems in human organs. In the mentioned studies, mostly work has been done with endoscopy and blood clot with simple Newtonian and non-Newtonian fluid models. In contrast, the present study deals with mass transport on particle-fluid motion through a ciliated annulus under different effects. Cilia motion plays a critical part, i.e., ciliary imperfections tend to create several human diseases. A genetic change compromises an appropriate function of the ciliopathies, cilia, which results in chronic disorders, i.e., primary ciliary dyskinesia and Senior-Loken syndrome or nephronophthisis. Furthermore, a flaw in primary cilium in renal tubule cells causes polycystic kidney disease. Ectopic pregnancy can occur due to a lack of functional cilia in a fallopian tube. If the cilia fail to move, then a fertilized ovum is unable to reach the uterus, which results in the ovum implant in a fallopian tube and tubal pregnancy will occur, which is the most usual type of ectopic pregnancy [29]. Therefore, the present study is essential to fill this gap and also beneficial to overcome the difficulties. Bi-viscosity fluid model is considered to examine the flow behavior. The governing mathematical modeling is performed under low Reynolds number approximation. Exact solutions are given for the fluid- and particulate-phase. The physical action of all the leading parameters is discussed against velocity, concentration, temperature profile, and the trapping mechanism is also presented through streamlines.

\section{Problem Formulation}

Consider two-dimensional co-axial infinite tubes. The outer tube is ciliated. The cylindrical coordinate system is selected, i.e., $\tilde{r}$ lies toward the radial direction, and $z$ lies toward the middle of an inner and the outer tube as given in Figure 2. The inner area between both the tubes is filled with bi-viscosity fluid. The flow is irrotational and the fluid is incompressible having constant viscosity. The fluid contains small spherical particles. The stress tensor for bi-viscosity fluid model [30] is defined as:

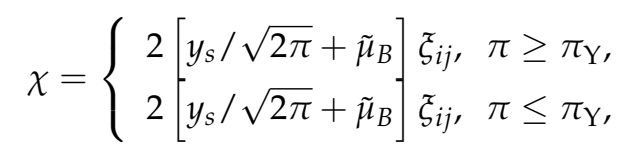

where $\tilde{\mu}_{B}$ the plastic viscosity, $\mathrm{Y}$ the volume fraction density, $\xi_{i j}$ the deformation rate of component, and $y_{s}$ the yield stress, $\pi$ denotes the second invariant tensor of $\xi_{i j}, \pi_{Y}$ represents the critical value comprises on the non-Newtonian fluid model. 


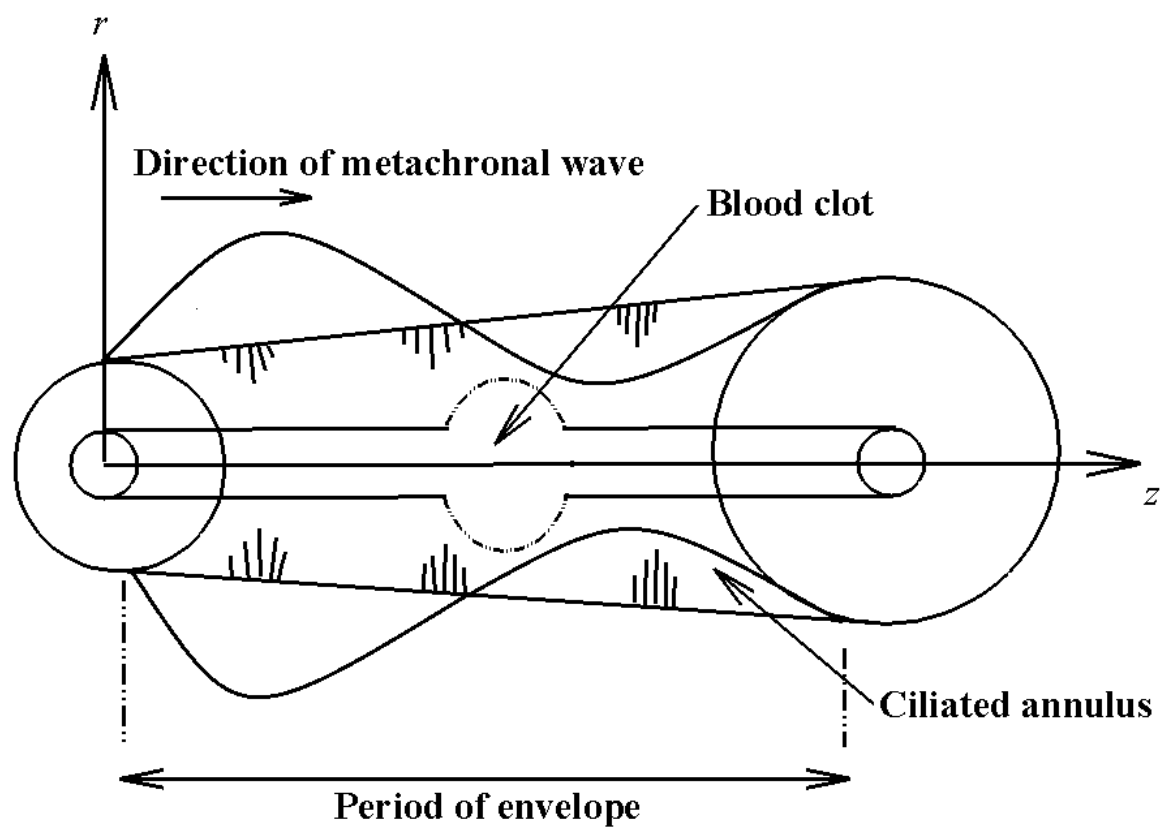

Figure 2. Blood flow structure through an ciliated annulus.

The Mathematical expression for the envelope of the cilia tips reads as [31,32]:

$$
\begin{aligned}
& \tilde{r}=\hbar_{1}=f_{1}(\tilde{t}, \tilde{z})=b_{0}+b_{0} \phi \cos [k(\tilde{z}-c \tilde{t})], \\
& \tilde{z}=\hbar_{2}=g_{1}(\tilde{t}, \tilde{z})=\tilde{z}_{0}+b_{0} \alpha \phi \sin [k(\tilde{z}-c \tilde{t})],
\end{aligned}
$$

where $k=2 \pi / \lambda, z_{0}$ describes the reference location of the cilia, the non-dimensional parameter $\phi$, which combines with $b_{0}$ (mean radius of the outer tube) in the form of $b_{0} \phi$ and represents the amplitude of metachronal wave, $\lambda$ is the metachronal wavelength, $c$ the velocity, and $\alpha$ describes the measure of the eccentricity of the elliptical motion.

The vertical and axial velocities are evaluated as [31,32]:

$$
\begin{gathered}
\tilde{u}=\frac{\partial \tilde{r}}{\partial \tilde{t}}=\frac{\partial f_{1}}{\partial \tilde{t}}+\frac{\partial f_{1}}{\partial \tilde{z}} \frac{\partial \tilde{z}}{\partial \tilde{t}}=\frac{\partial f_{1}}{\partial \tilde{t}}+\frac{\partial f_{1}}{\partial \tilde{z}} \tilde{u}, \tilde{z}=\tilde{z}_{0}, \\
\tilde{v}=\frac{\partial \tilde{z}}{\partial \tilde{t}}=\frac{\partial g_{1}}{\partial \tilde{t}}+\frac{\partial g_{1}}{\partial \tilde{z}} \frac{\partial \tilde{z}}{\partial \tilde{t}}=\frac{\partial g_{1}}{\partial \tilde{t}}+\frac{\partial g_{1}}{\partial \tilde{z}} \tilde{u}, \tilde{z}=\tilde{z}_{0},
\end{gathered}
$$

After some mathematical manipulation, Equations (4) and (5) read as:

$$
\begin{gathered}
\tilde{u}=-\frac{b_{0} \alpha c k \phi \cos [k(\tilde{z}-c \tilde{t})]}{1-b_{0} \alpha k \phi \cos [k(\tilde{z}-c \tilde{t})]}, \\
\tilde{v}=\frac{b_{0} c k \phi \sin [k(\tilde{z}-c \tilde{t})]}{1-b_{0} \alpha k \phi \cos [k(\tilde{z}-c \tilde{t})]} .
\end{gathered}
$$

The above boundary conditions help us to discriminate between the effective stroke and less effective recovery stroke of the cilia by considering the shortening of the cilia.

In view of above frame work, the mathematical modeling for the fluid-and particulate-phase is as follows [33]: 
(i) Fluid phase

The continuity and momentum equations are proposed as:

$$
\begin{gathered}
\varphi \frac{\partial \tilde{v}_{f}}{\partial \tilde{r}}+\varphi \frac{\tilde{v}_{f}}{\tilde{r}}+\varphi \frac{\partial \tilde{u}_{f}}{\partial \tilde{z}}=0 \\
\varphi \frac{\partial \tilde{p}}{\partial \tilde{r}}-C S\left(\tilde{v}_{p}-\tilde{v}_{f}\right)=\varphi\left[\frac{1}{\tilde{r}} \frac{\partial}{\partial \tilde{r}} r \chi_{\tilde{r} \tilde{r}}+\frac{\partial}{\partial \tilde{z}} \chi_{\tilde{r} \tilde{z}}-\frac{\chi_{\tilde{\theta} \tilde{\theta}}}{\tilde{r}}\right], \\
\varphi \frac{\partial \tilde{p}}{\partial \tilde{z}}-C S\left(\tilde{u}_{p}-\tilde{u}_{f}\right)=\varphi\left[\frac{\partial}{\partial \tilde{z}} \chi_{\tilde{z} \tilde{z}}+\frac{1}{\tilde{r}} \frac{\partial}{\partial \tilde{r}} \tilde{r} \chi_{\tilde{r} \tilde{z}}\right],
\end{gathered}
$$

The energy equation for the current flow is described as

$$
\varphi \rho_{f} \tilde{c}\left[\frac{\partial}{\partial \tilde{t}}+\mathbf{V}_{f} \cdot \nabla\right] T_{f}=\kappa \varphi \nabla^{2} T_{f}+\varphi \chi_{\tilde{r} \tilde{z}}\left[\frac{\partial u_{f}}{\partial \tilde{r}}\right]+\frac{\rho_{p} c_{p} C}{\omega_{T}}\left(T_{p}-T_{f}\right)
$$

The concentration equation for the current flow is described as

$$
\varphi\left[\frac{\partial}{\partial \tilde{t}}+\mathbf{V}_{f} \cdot \nabla\right] K_{f}=D_{m} \varphi \nabla^{2} K_{f}+\frac{\rho_{p} C}{\rho_{f} \omega_{c}}\left(\varphi_{p}-\varphi_{f}\right)+\frac{D_{m}}{T_{m}} \varphi K_{T} \nabla^{2} T_{f}
$$

where $\varphi=1-C$.

(ii) Particulate phase

The continuity and momentum equation for this case read as

$$
\begin{gathered}
C \frac{\partial \tilde{v}_{p}}{\partial \tilde{r}}+C \frac{\tilde{v}_{p}}{\tilde{r}}+C \frac{\partial \tilde{u}_{p}}{\partial \tilde{z}}=0, \\
C \frac{\partial \tilde{p}}{\partial \tilde{r}}-S C\left(\tilde{v}_{f}-\tilde{v}_{p}\right)=0 \\
C \frac{\partial \tilde{p}}{\partial \tilde{z}}-S C\left(\tilde{u}_{f}-\tilde{u}_{p}\right)=0
\end{gathered}
$$

In this case, the energy equation is described as

$$
\rho_{p} C c_{p}\left[\frac{\partial}{\partial \tilde{t}}+\mathbf{V}_{p} \cdot \nabla\right] T_{p}=\frac{\rho_{p} c_{p} C}{\omega_{T}}\left(T_{f}-T_{p}\right),
$$

The concentration equation is described as

$$
\left[\frac{\partial}{\partial \tilde{t}}+\mathbf{V}_{p} \cdot \nabla\right] K_{p}=\frac{1}{\omega_{c}}\left(K_{f}-K_{p}\right)
$$

where $S$ the drag coefficient, $\rho$ the density of the fluid, $C$ the particle volume fraction density, $T$ the temperature, $\omega_{T}$ the thermal equilibrium time, $\omega_{c}$ is the required time period by a particle to regulate its concentration associated to the fluid, $D_{m}$ the mass diffusivity coefficient, $K_{T}$ is the thermal diffusion ratio, $T_{m}$ the mean temperature, $\kappa$ the thermal conductivity, $c_{p}$ particle-phase specific heat, and $\tilde{c}$ the specific heat at constant volume. 
The mathematical form of drag coefficient is expressed as [23]

$$
S=\frac{9}{2} \frac{\mu_{0}}{B_{0}^{2}} \Phi(C), \Phi(C)=\frac{3 C+4+[C(8-3 C)]^{1 / 2}}{(2-3 C)^{2}}
$$

where $B_{0}$ is the radius of each particle, and $\mu_{0}$ the fluid viscosity. The empirical relation for the viscosity suspension is expressed as [23]

$$
\tilde{m}=0.70 e^{\left[\frac{1107}{T} e^{(-1.69 C)}+2.49 C\right]}, \mu_{s}=\frac{\mu_{0}}{1-\tilde{m} C^{\prime}}
$$

where $\mu_{s}$ denotes the viscosity of particle fluid mixture.

It is noted here that the results reduced for dusty-gas flows for small particle volume fraction as presented by Marble [34].

Defining the following non-dimensional variables

$$
\begin{gathered}
r=\frac{\tilde{r}}{b_{0}}, u_{f, p}=\frac{\tilde{u}_{f, p}}{c}, z=\frac{\tilde{z}}{\lambda}, v_{f, p}=\frac{\lambda \tilde{v}_{f, p}}{b_{0} c}, t=\frac{\tilde{t} c}{\lambda}, p=\frac{b_{0}^{2}}{\lambda \mu_{0} c} \tilde{p}, \bar{\mu}=\frac{\mu_{s}}{\mu_{0}}, \\
v_{0}=\frac{\tilde{v}_{0}}{c}, r_{1}=\frac{\tilde{r}_{1}}{b_{0}}, r_{2}=\frac{\tilde{r}_{2}}{b_{0}}, \theta_{f, p}=\frac{T_{f, p}-T_{0}}{T_{1}-T_{0}}, \vartheta_{f, p}=\frac{K_{f, p}-K_{0}}{K_{1}-K_{0}} .
\end{gathered}
$$

Applying Equation (20) in Equations (8)-(18), and applying the approximation of low Reynolds number and ignoring the inertial forces. The resulting equations are found as

$$
\begin{gathered}
0=\frac{\partial p}{\partial r} \\
\frac{\partial p}{\partial z}=\frac{C S b_{0}^{2}}{\varphi \mu_{0}}\left(u_{p}-u_{f}\right)+\frac{\bar{\mu}}{r} \eta \frac{\partial}{\partial r}\left(r \frac{\partial u_{f}}{\partial r}\right) .
\end{gathered}
$$

It is noted here that the results for Newtonian fluid model can be recovered by taking $\zeta \rightarrow \infty$. The temperature and concentration equations read as

$$
\begin{gathered}
\frac{1}{r} \frac{\partial}{\partial r}\left(r \frac{\partial \theta_{f}}{\partial r}\right)+B_{n} \bar{\mu} \eta\left(\frac{\partial u_{f}}{\partial r}\right)^{2}=0, \\
\frac{1}{r} \frac{\partial}{\partial r}\left(r \frac{\partial \vartheta_{f}}{\partial r}\right)+S_{c} S_{r} \frac{1}{r} \frac{\partial}{\partial r}\left(r \frac{\partial \theta_{f}}{\partial r}\right)=0,
\end{gathered}
$$

where $\eta=(1+1 / \zeta), B_{n}$ the Brinkman number, $\delta$ defines the wave number, $S_{c}$ the Schmidt number, Pr the Prandtl number, $S_{r}$ the Soret number, Ec the Eckert number, and $\zeta$ the fluid parameter. These parameters are defined as

$$
\begin{aligned}
& B_{n}=\operatorname{EcPr}, \operatorname{Pr}=\frac{\tilde{c} v \rho_{f}}{\kappa}, \zeta=\frac{\tilde{\mu}_{B} \sqrt{2 \pi_{Y}}}{y_{s}}, \mathrm{Ec}=\frac{c^{2}}{\tilde{c}\left(T_{1}-T_{0}\right)}, S_{c}=\frac{v}{D_{m}}, \delta=\frac{b_{0}}{\lambda}, \\
& S_{r}=\frac{D_{m} K_{T}}{v T_{m}}\left(\frac{\theta_{1}-\theta_{0}}{\vartheta_{1}-\vartheta_{0}}\right) .
\end{aligned}
$$

The particulate-phase equations are found as

$$
\frac{\partial p}{\partial z}+\frac{S b_{0}^{2}}{\mu_{0}}\left(u_{p}-u_{f}\right)=0
$$




$$
\begin{aligned}
& \theta_{f}=\theta_{p}, \\
& \vartheta_{f}=\vartheta_{p} .
\end{aligned}
$$

From Equation (21), it is found that $p$ cannot be the function of $r$. The relevant boundary conditions read as

$$
\begin{gathered}
u_{f}\left(r_{1}\right)=v_{0}, \theta_{f}\left(r_{1}\right)=1, \quad r_{1}=a(z)=a_{0}+h_{c} e^{-\pi^{2}\left(z-z_{d}-0.5\right)^{2},} \\
u_{f}\left(r_{2}\right)=-\frac{2 \pi \alpha \phi \delta \cos 2 \pi \Xi}{1-2 \pi \alpha \phi \delta \cos 2 \pi \Xi}, \theta_{f}\left(r_{2}\right)=0, \quad r_{2}=1+\frac{\lambda \Gamma z}{b_{0}}+\phi \sin 2 \pi \Xi,
\end{gathered}
$$

where $\Xi=(z-t), \Gamma$ is a constant which represents the magnitude that relies on the annulus length and its exit inlet dimensions, maximum height of the clot denoted by $h_{\mathcal{c}}, v_{0}$ typify the velocity of the inner tube, the axial displacement of the clot is denoted by $z_{d}$, and the radius of the inner tube which makes the clot in the appropriate place is denoted by $a_{0}$. The results for endoscopy can be reduced by considering $h_{c}=0$ in Equation (31) as a particular case of the present study.

\section{Solutions of the Proposed Problem}

Equations (22)-(24) are solved analytically using a computational software "Mathematica 10.3v", and the exact solutions are presented below:

$$
\begin{gathered}
u_{f}=C_{1}+r C_{2}+C_{3} r \log r, \\
u_{p}=C_{1}+r C_{2}+C_{3} r \log r-\frac{\mu_{0}}{S b_{0}^{2}} \frac{d p}{d z},
\end{gathered}
$$

The solutions for the temperature profile for particulate- and fluid-phase are found as

$$
\theta_{f, p}=\theta_{0}+r^{2} \theta_{1}+r^{4} \theta_{2}+\theta_{3} \log r+\theta_{4} \log ^{2} r,
$$

The solutions for the concentration profile for particulate- and fluid-phase are found as

$$
\vartheta_{f, p}=\vartheta_{0}+r^{2} \vartheta_{1}+r^{4} \vartheta_{2}+\vartheta_{3} \log r+\vartheta_{4} \log ^{2} r+\vartheta_{5} \log ^{3} r,
$$

and the constants appearing in above Equations (31)-(34) i.e. $C_{n}, \theta_{n}, \vartheta_{n}(n=1,2 \ldots)$ are given Appendix A.

The instantaneous volume flow rate for the present flow read as

$$
Q(t, z)=2 \pi \varphi \int_{r_{1}}^{r_{2}} r u_{f} \mathrm{~d} r+2 \pi C \int_{r_{1}}^{r_{2}} r u_{p} \mathrm{~d} r .
$$

The pressure gradient can be obtained after solving the above equation.

The pressure rise along the whole ciliated annulus can be determined as

$$
\Delta p=\int_{0}^{L / \lambda} \wp \mathrm{d} z
$$

where $\wp$ represents the pressure gradient. 


\section{Graphical Analysis}

In thissection, using the obtained numerical results we analyze the behavior of all the physical parameter. Particularly, we determine the behavior of velocity profile, concentration, and the temperature profile, against the height of the clot $h_{c}$, particle volume fraction $C$, wave number $\delta$, Soret number $S_{r}$, Brinkmann number $B_{n}$, Schmidt number $S_{c}$, and eccentricity of the elliptic path of cilia $\alpha$. Following parametric values [1] are chosen to analyze the graphical performance of all the leading parameters, i.e., $b_{0}=1.25 \mathrm{~cm}, \phi=0.1-0.5, C=0-0.6, \alpha=0.3-1, \Gamma=3 b_{0} / \lambda$, $L=\lambda=8.01 \mathrm{~cm}, \delta=0.05-0.2$. Furthermore, the results for single-phase model can be recovered by considering $C=0$ in the governing equations (see Equations (21)-(28)). Assume that the instantaneous volume flow rate is periodic in $\Xi$, i.e.,

$$
\frac{Q}{\pi}=-\frac{\phi^{2}}{2}+\frac{\bar{Q}}{\pi}+2 \phi \sin 2 \pi \Xi+\frac{2 \phi \lambda z}{b_{0}} \Gamma \sin 2 \pi \Xi+\phi^{2} \sin ^{2} 2 \pi \Xi,
$$

where $\bar{Q}$ denotes the average time flow rate over one period of wavelength.

Figure 3 depicts the behavior of blood clots and particle volume fraction on the velocity profile. We can observe from this figure that an increment in particle volume fraction $C$ significantly suppresses the velocity profile. The velocity profile shows a decreasing behavior for endoscopic case, i.e., $h_{c}=0$, whereas it increases due to the blood clot $h_{c}=0.15$. It can be observed from Figure 4 that both parameters $\alpha$ and $\delta$ cause a positive impact on the velocity profile while its trend becomes reverse when $r>1.35$. Figure 5 shows a plot of velocity profile against numerous values of $\phi$. It can be seen from this figure that the velocity profile is remarkably suppressed with increments in $\phi$. Furthermore, we also noticed that as compared with non-Newtonian case $\zeta=0.1$, the fluid velocity lessen more when the fluid behaves as a Newtonian model $\zeta \rightarrow \infty$.

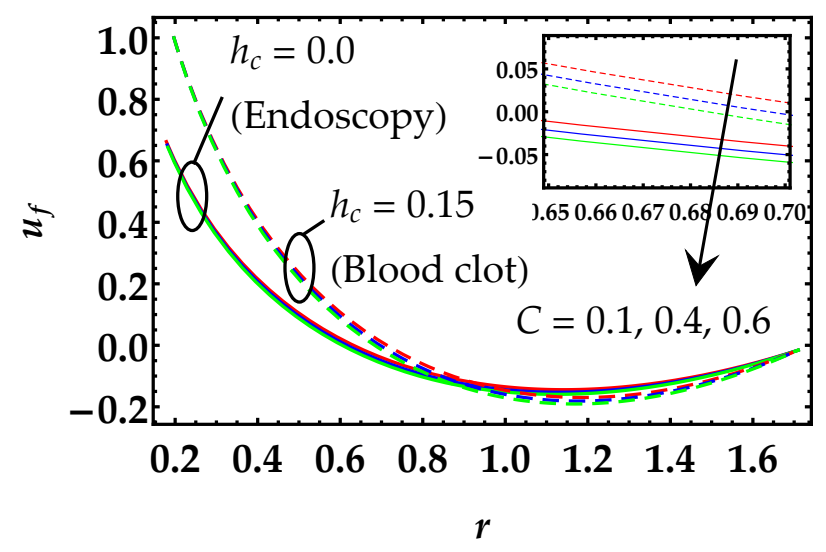

Figure 3. Velocity curves for different values of $h_{c}$ and $C$.

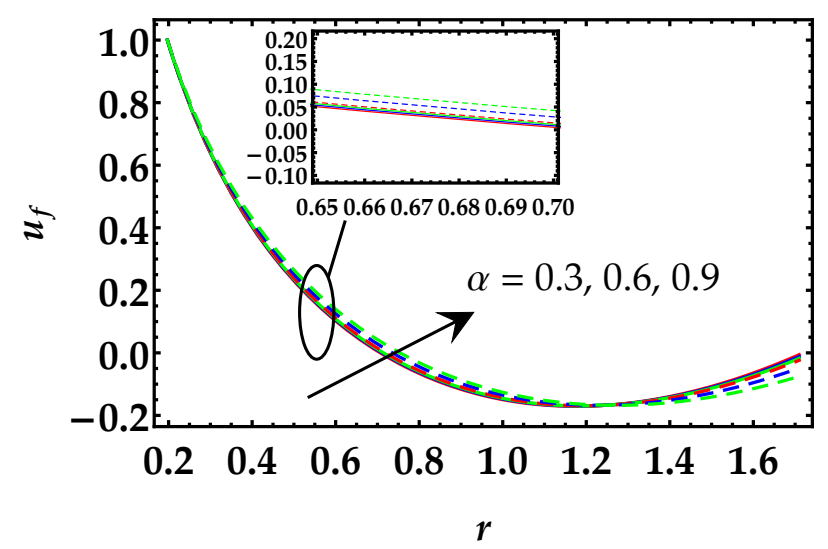

Figure 4. Velocity curves for different values of $\alpha$ and $\delta$. Solid line: $\delta=0.05$, dashed line: $\delta=0.2$. 


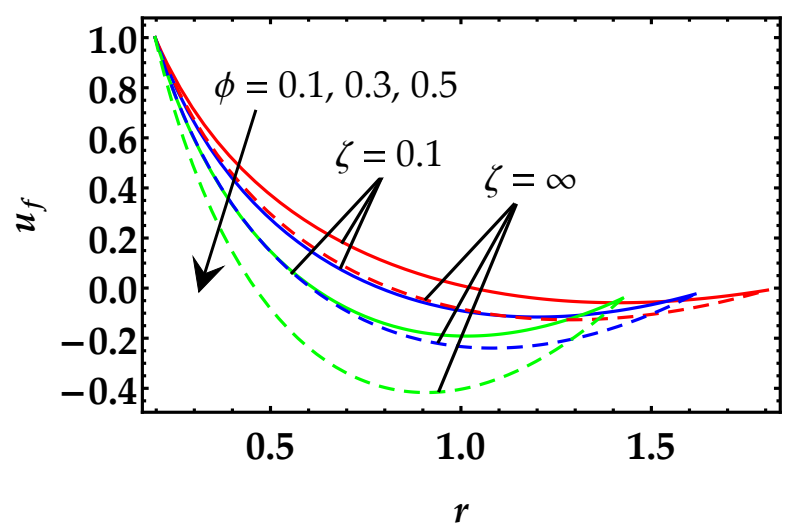

Figure 5. Velocity curves for different values of $\phi$ and $\zeta$.

In Figures 6-8 we see the mechanism of temperature profile plotted against the multiple leading parameters. From Figure 6, we can see that the temperature profile rises due to the increment in particle volume fraction $C$. Further, we noticed that for the blood clot case $h_{c}=0.15$, the temperature profile is increasing and has a higher magnitude as compared with the endoscopic case $h_{c}=0$. It is analyzed from Figure 7 that the parameters $\alpha$ and $\delta$ restrain the temperature profile. Unfortunately, both parameters have small effects, especially when the wavenumber is very small at $\delta=0.05$. Figure 8 shows plots with multiple values of Brinkman number $B_{n}$. Brinkman number represents the product of Eckert and Prandtl number Ec $\times$ Pr. Generally, it is the ratio between heat generated due to viscous dissipation and transport of heat due to molecular conduction. It can noticed that the temperature profile remarkably increases for higher values of Brinkman number. However, a similar behavior is observed against the higher values of $\phi$.

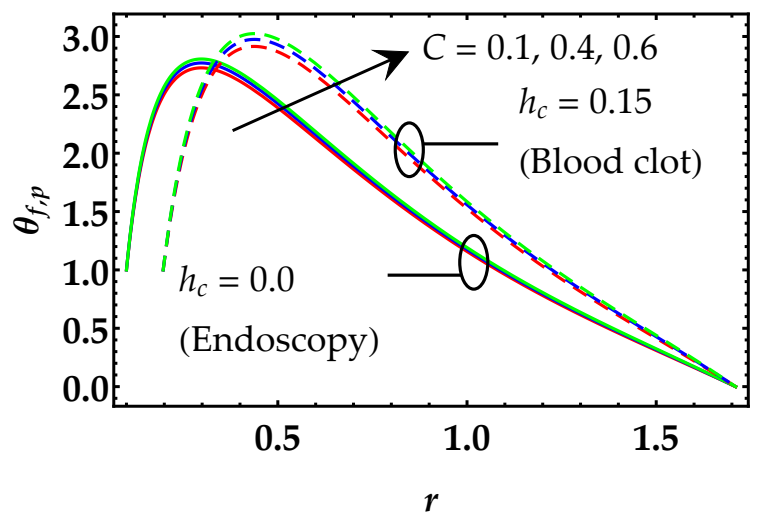

Figure 6. Temperature distribution for different values of $h_{\mathcal{c}}$ and $C$.

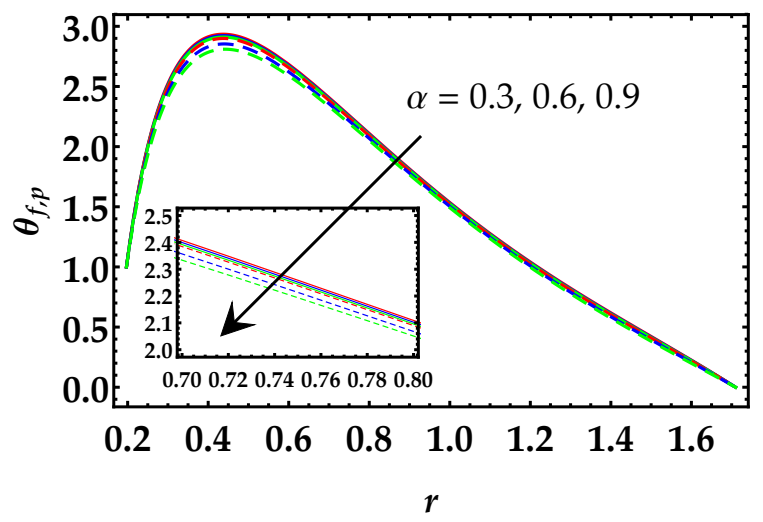

Figure 7. Temperature distribution for different values of $\alpha$ and $\delta$. Solid line: $\delta=0.05$, dashed line: $\delta=0.2$. 


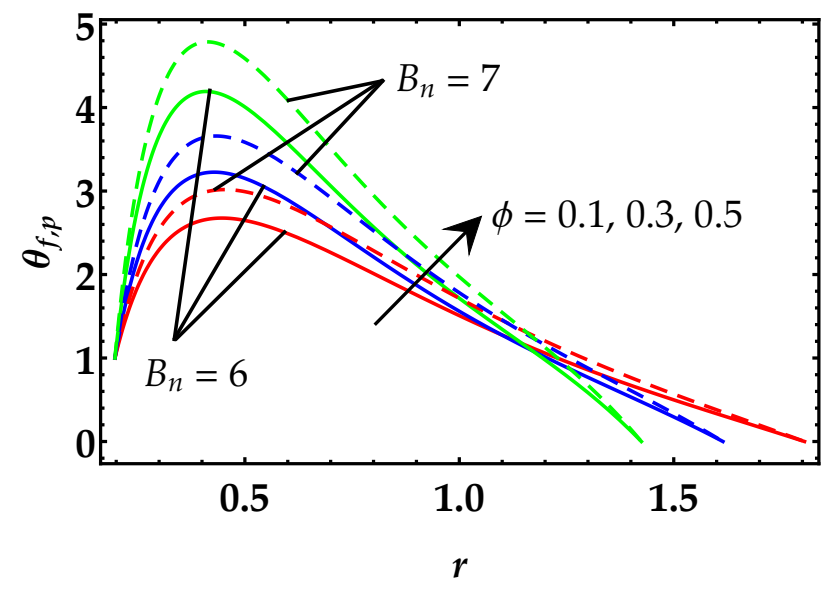

Figure 8. Temperature distribution for different values of $\phi$ and $B_{n}$.

Figure 9 is illustrated to analyze the mechanisms of Schmidt number $S_{c}$ and Soret number $S_{r}$ on the concentration profile. We can see from this figure that the concentration profile shows a decreasing mechanism against both parameters and remains uniform throughout the entire region. An increment in Schmidt number indicates that the viscous diffusion rate is more dominant as compared with the molecular diffusion rate, whose results tend to decline the concentration profile. Similarly, when the Soret number increases, the Thermophoresis forces generated, which oppose the concentration profile.

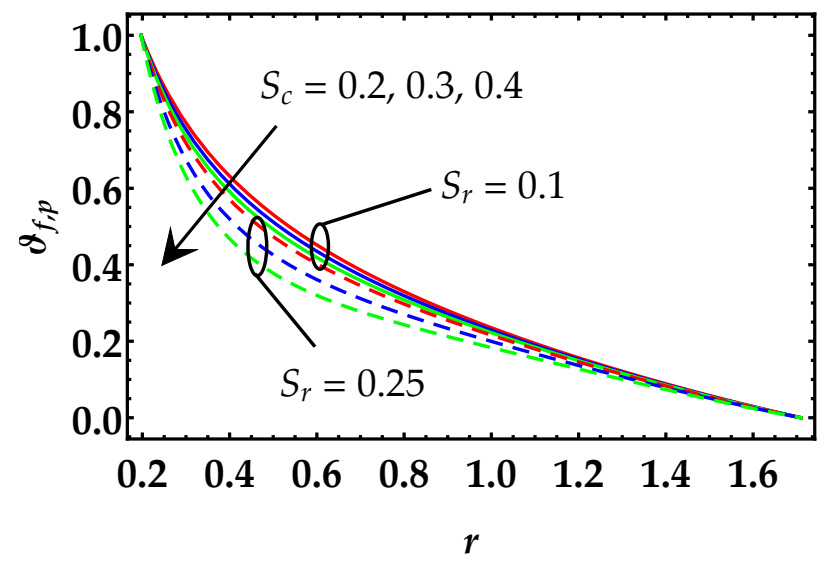

Figure 9. Concentration distribution for different values of $S_{c}$ and $S_{r}$.

Figures 10-12 depict the variation of pressure rise versus time against different values of emerging parameters. It can be observed from Figure 10 that by enhancing the particle volume fraction $C$, the pressure rise is significantly decreasing, while due to the presence of blood clot, more pressure is required to pass the fluid. Further, we can see that the pressure rise is maximum in the region when $t \in(0.3,0.7)$. It is clear from the Figure 11 that both parameters $\alpha$ and $\delta$ reveal versatile behavior on the pressure rise. We can also see that there are two critical points, for instance, at $t=0.4$ and $t=0.9$. In the region $t \in(0.4,0.9)$ the pressure rise acts as an increasing function whereas in the other area it decreases. Similarly, we can observe that the pressure rise increases due to the increment in $\phi$, as shown in Figure 12.

Trapping mechanism is presented in Figures $13-15$ for different values of $\alpha, \delta$ and $h_{c}$. It can be noticed from Figure 13 that by increasing the values of $\alpha$, the trapping bolus reduces, and a number of boluses disappear. Similarly, in Figure 14, we can see that the higher values of $\delta$ tend to diminish the immensity of the trapping bolus, whereas the number of trapping bolus increase and streamlines increases. It is seen in Figure 15 that when the height of the blood clot increases, then streamlines shrink, and trapping bolus increase significantly. 


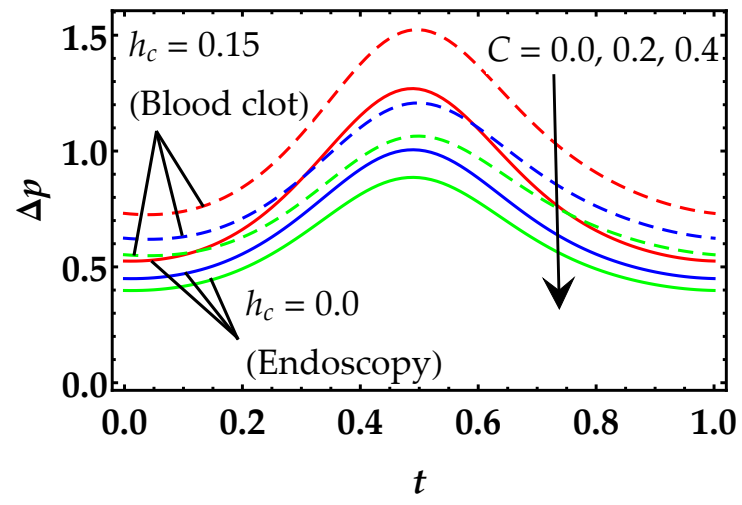

Figure 10. Pressure rise for different values of $h_{c}$ and $C$.

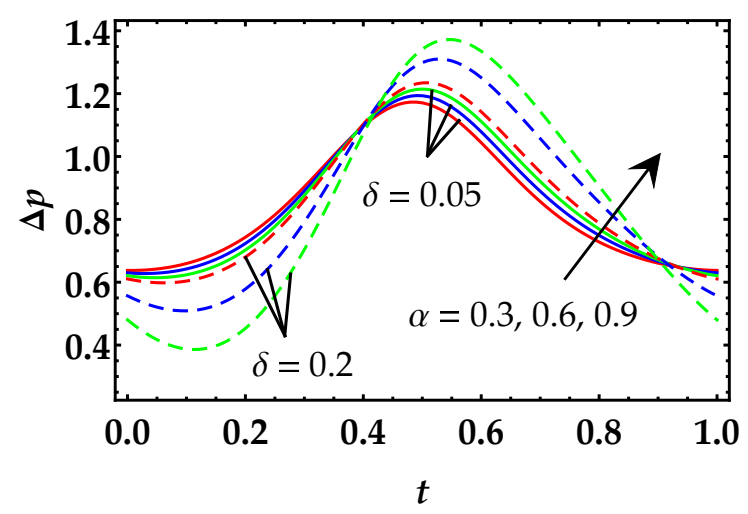

Figure 11. Pressure rise for different values of $\alpha$ and $\delta$.

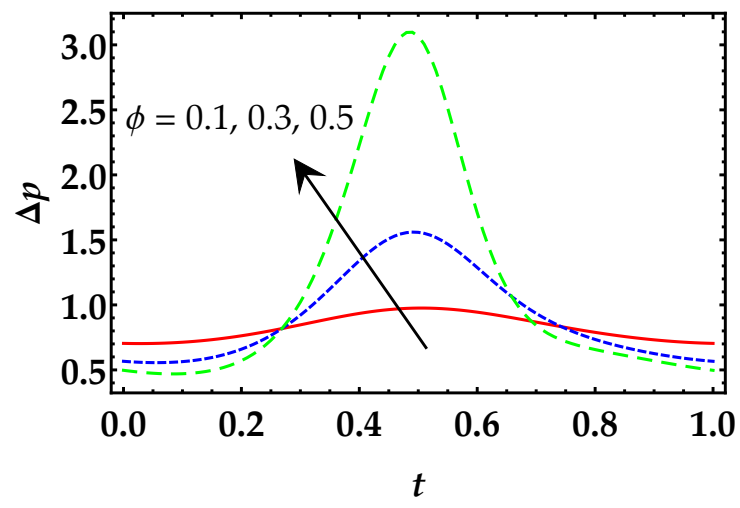

Figure 12. Pressure rise for different values of $\phi$.

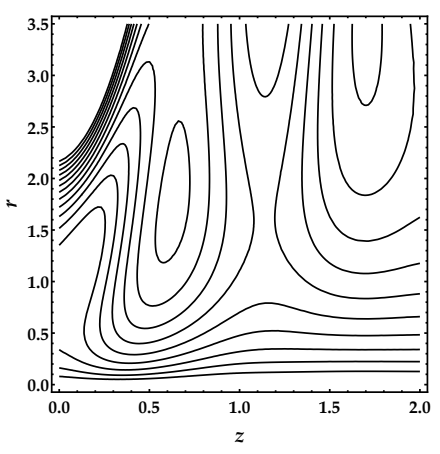

(a) $\alpha=0.3$

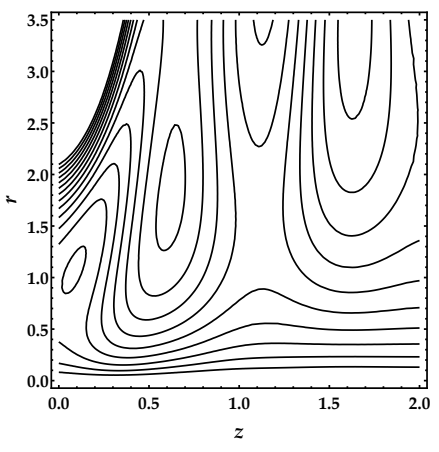

(b) $\alpha=0.6$

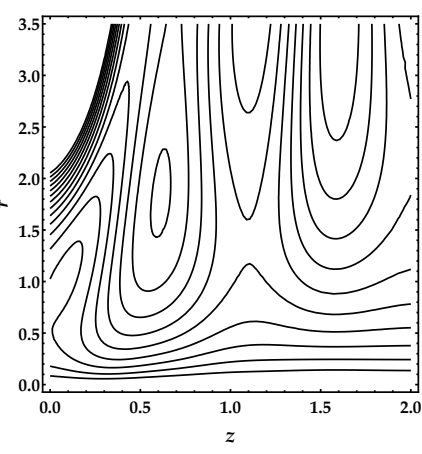

(c) $\alpha=0.9$

Figure 13. Trapping mechanism for different values of $\alpha$. 


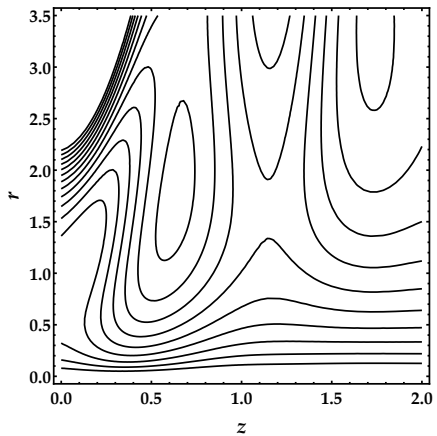

(a) $\delta=0.05$

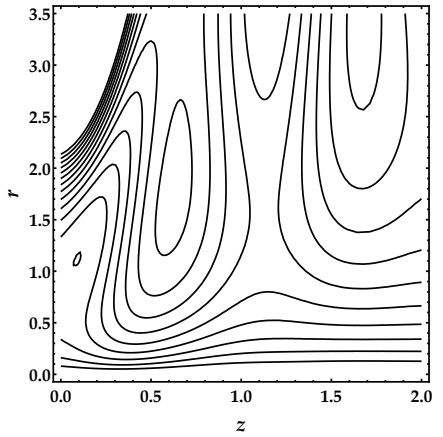

(b) $\delta=0.1$

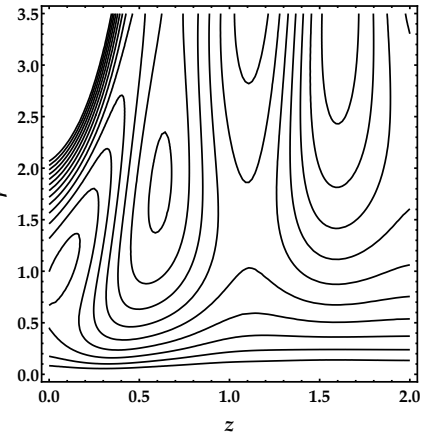

(c) $\delta=0.2$

Figure 14. Trapping mechanism for different values of $\delta$.

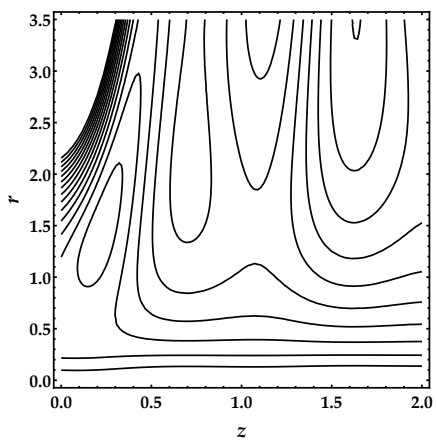

(a) $h_{c}=0$

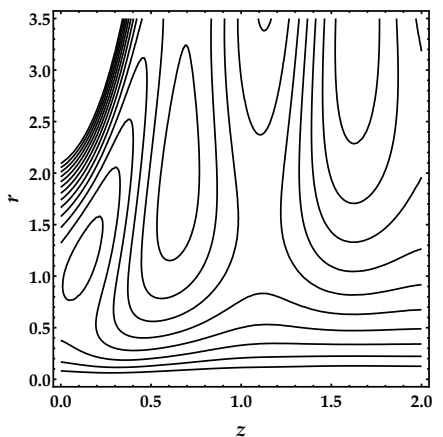

(b) $h_{c}=0.1$

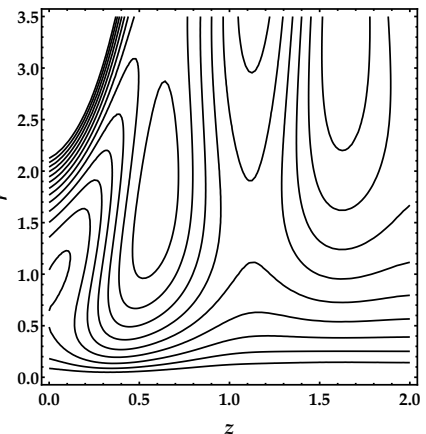

(c) $h_{c}=0.2$

Figure 15. Trapping mechanism for different values of $h_{c}$.

\section{Conclusions}

In this study, we explained the behavior of particle-fluid with mass and heat transfer through a ciliated annulus. The effects of endoscopy and blood clot are also taken into account. To analyze the behavior of fluid in an annulus, we considered the bi-viscosity fluid model. The mathematical formulation is undertaken for low Reynolds number approximation. The formulated differential equations are analytically solved, and closed-form solutions are presented. The main observations of the present study are followed as:

(i) It is noticed that particle volume strongly opposes the flow, whereas the fluid velocity also decreases for the endoscopic case as compared with the blot clot case.

(ii) Velocity of the fluid also rises due to the enhancement in $\alpha$ and $\delta$.

(iii) Temperature profile shows a higher magnitude in the presence of solid particles, while similar behavior is noticed during blood clot.

(iv) Brinkman number shows the dominant behavior on the temperature profile and enhances the temperature profile remarkably.

(v) Concentration profile reveals a decreasing behavior with the increase in the values of Soret and Schmidt numbers.

(vi) $\alpha$ and $\delta$ depict versatile behavior on the pressure rise profile.

(vii) Particle volume fraction opposes the pressure rise, whereas the blood clot enhances the pressure rise.

(viii) Trapping mechanism shows that the number of bolus gets bigger, and the streamlines gather as the height of the blood clot increases.

Furthermore, in this study, several effects have been ignored, i.e., magnetic field, porosity, chemical reaction and activation energy, respectively, which can be considered in future research. 
Author Contributions: Investigation, M.M.B.; Methodology \& Conceptualization, R.E.; Validation, A.F.E.; Writing-review \& editing, S.M.S. All authors have read and agreed to the published version of the manuscript.

Acknowledgments: Authors thanks to those who are working in front line to save the humanity from corona pandemic in China and across the globe. We appreciate their devotion and services.

Funding: This research received no external funding.

Conflicts of Interest: The authors declare no conflict of interest.

\section{Appendix A}

$$
\begin{aligned}
& C_{1}=\frac{1}{4 \varphi \eta} \frac{d p}{d z} \\
& C_{2}=\frac{1}{4 \varphi \eta}\left[\frac{d p}{d z}\left(r_{2}^{2}-r_{1}^{2}\right)-4 \xi \varphi \eta\right],, \\
& C_{3}=\frac{\theta_{5}}{4 \varphi \eta \zeta \log \frac{r_{1}}{r_{2}}}, \\
& \xi=-\frac{2 \pi \alpha \varepsilon \delta \cos 2 \pi \Xi}{1-2 \pi \alpha \varepsilon \delta \cos 2 \pi \Xi} . \\
& \theta_{0}=\frac{1}{16 \theta_{7}^{2} \log \frac{r_{1}}{r_{2}}}\left[-\left\{16 \theta_{2}^{2}+B_{n} \theta_{6} r_{1}^{2}\left(8 \theta_{5}+\theta_{6} r_{1}^{2}\right) \eta\right\}-8 B_{n} \theta_{5}^{2} \eta \log r_{1}^{2} \log r_{2}\right. \\
& \left.+B_{n} \eta \log r_{1}\left(\theta_{6} r_{2}^{2}\left(8 \theta_{5}+\theta_{6} r_{2}^{2}\right)+8 \theta_{5}^{2} \log ^{2} r_{2}\right)\right], \\
& \theta_{1}=-\frac{8 B_{n} \theta_{5} \theta_{6} \eta}{16 \theta_{7}^{2}} \\
& \theta_{2}=-\frac{B_{n} \theta_{6}^{2} \eta}{16 \theta_{7}^{2}} \\
& \theta_{3}=\frac{1}{16 \theta_{7}^{2} \log \frac{r_{1}}{r_{2}}}\left[16 \theta_{7}^{2}+B_{n} \theta_{6}\left(r_{1}^{2}-r_{2}^{2}\right)\left\{8 \theta_{5}+\theta_{6}\left(r_{1}^{2}+r_{2}^{2}\right)\right\} \eta+8 B_{n} \theta_{5}^{2} \eta \log ^{2} \frac{r_{1}}{r_{2}}\right] \\
& \theta_{4}=-\frac{B_{n} \theta_{5}^{2} \eta}{2 \theta_{7}^{2}} \\
& \theta_{5}=\zeta\left(\frac{d p}{d z}\left(r_{1}^{2}-r_{2}^{2}\right)+\left(\xi-v_{0}\right) 4 \varphi \eta\right) \\
& \theta_{6}=2 \zeta \frac{d p}{d z} \log \frac{r_{2}}{r_{1}}
\end{aligned}
$$




$$
\begin{aligned}
& \theta_{7}=4 \varphi \eta \zeta \log \frac{r_{2}}{r_{1}} \\
& \vartheta_{0}=\frac{1}{192 \theta_{7}^{2} \log \frac{r_{1}}{r_{2}}}\left[3\left(-64 \theta_{7}^{2}+B_{n} \theta_{6} r_{1}^{2}\left(16 \theta_{5}+3 \theta_{6} r_{1}^{2}\right) S_{c} S_{r} \eta\right) \log r_{2}\right. \\
& +96 B_{n} \theta_{5}^{2} S_{c} S_{r} \eta \log ^{2} r_{1} \log r_{2}+16 B_{n} \theta_{5}^{2} S_{c} S_{r} \eta \log r_{1}^{3} \log r_{2} \\
& +S_{c} S_{r} \log r_{1}\left\{-3 B_{n} \theta_{5} r_{2}^{2}\left(16 \theta_{5}+3 \theta_{6} r_{2}^{2}\right) \eta+2 \log r_{2}\left(48 \theta_{7}^{2}+3 B_{n}\right.\right. \\
& \left.\left.\left.\times \theta_{6}\left(r_{1}^{2}-r_{2}^{2}\right)\left(8 \theta_{5}+\theta_{6}\left(r_{1}^{2}+r_{2}^{2}\right)\right) \eta-8 B_{n} \theta_{5}^{2} \eta \log r_{2}\left(6+\log r_{2}\right)\right)\right\}\right], \\
& \vartheta_{1}=\frac{B_{n} S_{c} S_{r} \theta_{5} \theta_{6} \eta}{\theta_{7}^{2}} \\
& \vartheta_{2}=\frac{3 B_{n} S_{c} S_{r} \theta_{6}^{2} \eta}{64 \theta_{7}^{2}} \\
& \vartheta_{3}=\frac{1}{192 \theta_{7}^{2} \log \frac{r_{1}}{r_{2}}}\left[192 \theta_{7}^{2}-3 B_{n} \theta_{5}\left(r_{1}^{2}-r_{2}^{2}\right)\left(16 \theta_{5}+3 \theta_{6}\left(r_{1}^{2}+r_{2}^{2}\right)\right) S_{c} S_{r} \eta\right. \\
& +2 S_{c} S_{r}\left\{\operatorname { l o g } r _ { 1 } \left(-48 \theta_{7}^{2} \zeta-3 B_{n} \theta_{6}\left(r_{1}^{2}-r_{2}^{2}\right)\left(8 \theta_{5}+\theta_{6}\left(r_{1}^{2}+r_{2}^{2}\right)\right) \eta-8 B_{n}\right.\right. \\
& \left.\theta_{5}^{2} \eta \log r_{1}\left(6+\log r_{1}\right)\right) 3 \zeta\left(16 \theta_{7}^{2}+B_{n} \theta_{6}\left(r_{1}^{2}-r_{2}^{2}\right)\left(8 \theta_{5}+\theta_{6}\left(r_{1}^{2}+r_{2}^{2}\right)\right) \eta\right. \\
& \left.\left.\left.+8 B_{n} \theta_{5}^{2} \eta \log r_{1}^{2}\right) \log r_{2}+24 B_{n} \theta_{5}^{2} \eta\left(2+\log r_{1}\right) \log r_{2}^{2}+8 B_{n} \theta_{5}^{2} \eta \log r_{2}^{3}\right\}\right], \\
& \vartheta_{4}=\frac{1}{32 \theta_{7}^{2} \log \frac{r_{1}}{r_{2}}}\left[S _ { c } S _ { r } \left\{16 \theta_{7}^{2}+B_{n} \theta_{6}\left(r_{1}^{2}-r_{2}^{2}\right)\left(8 \theta_{5}+\theta_{6}\left(r_{1}^{2}+r_{2}^{2}\right)\right) \eta\right.\right. \\
& \left.\left.+8 B_{n} \theta_{5}^{2} \eta \log \frac{r_{1}}{r_{2}}\left(2+\log r_{1}+\log r_{2}\right)\right\}\right], \\
& \vartheta_{5}=-\frac{B_{n} \theta_{5}^{2} S_{c} S_{r}}{6 \theta_{7}^{2}} \eta
\end{aligned}
$$

\section{References}

1. Lardner, T.; Shack, W. Cilia transport. Bull. Math. Biophys. 1972, 34, 325-335. [CrossRef] [PubMed]

2. Nadeem, S.; Munim, A.; Shaheen, A.; Hussain, S. Physiological flow of Carreau fluid due to ciliary motion. AIP Adv. 2016, 6, 035125. [CrossRef]

3. Nadeem, S.; Sadaf, H. Ciliary motion phenomenon of viscous nanofluid in a curved channel with wall properties. Eur. Phys. J. Plus 2016, 131, 65. [CrossRef]

4. Maiti, S.; Pandey, S. Rheological fluid motion in tube by metachronal waves of cilia. Appl. Math. Mech. 2017, 38, 393-410. [CrossRef]

5. Abo-Elkhair, R.; Mekheimer, K.S.; Moawad, A. Cilia walls influence on peristaltically induced motion of magneto-fluid through a porous medium at moderate Reynolds number: Numerical study. J. Egypt. Math. Soc. 2017, 25, 238-251. [CrossRef]

6. Bhatti, M.; Zeeshan, A.; Rashidi, M. Influence of magnetohydrodynamics on metachronal wave of particle-fluid suspension due to cilia motion. Eng. Sci. Technol. Int. J. 2017, 20, 265-271. [CrossRef] 
7. Ashraf, H.; Siddiqui, A.M.; Rana, M.A. Fallopian tube assessment of the peristaltic-ciliary flow of a linearly viscous fluid in a finite narrow tube. Appl. Math. Mech. 2018, 39, 437-454. doi:10.1007/s10483-018-2305-9. [CrossRef]

8. Ramesh, K.; Tripathi, D.; Bég, O.A. Cilia-assisted hydromagnetic pumping of biorheological couple stress fluids. Propuls. Power Res. 2019, 8, 221-233. [CrossRef]

9. Soo, S.L. Particulates And Continuum-Multiphase Fluid Dynamics: Multiphase Fluid Dynamics; Routledge: Abingdon, UK, 2018.

10. Hu, H.H.; Joseph, D.D.; Crochet, M.J. Direct simulation of fluid particle motions. Theor. Comput. Fluid Dyn. 1992, 3, 285-306. [CrossRef]

11. Feng, J.; Huang, P.; Joseph, D. Dynamic simulation of sedimentation of solid particles in an Oldroyd-B fluid. J. Non-Newton. Fluid Mech. 1996, 63, 63-88. [CrossRef]

12. Lima, R.; Ishikawa, T.; Imai, Y.; Takeda, M.; Wada, S.; Yamaguchi, T. Radial dispersion of red blood cells in blood flowing through glass capillaries: the role of hematocrit and geometry. J. Biomech. 2008, 41, 2188-2196. [CrossRef] [PubMed]

13. Mekheimer, K.S.; Abd Elmaboud, Y. Peristaltic transport of a particle-fluid suspension through a uniform and non-uniform annulus. Appl. Bion. Biomech. 2008, 5, 47-57. [CrossRef]

14. Mekheimer, K.S.; Mohamed, M.S. Peristaltic transport of a pulsatile flow for a particle-fluid suspension through a annular region: Application of a clot blood model. Int. J. Sci. Eng. Res. 2014, 5, 849-859.

15. Bhatti, M.; Zeeshan, A.; Ijaz, N. Slip effects and endoscopy analysis on blood flow of particle-fluid suspension induced by peristaltic wave. J. Mol. Liquids 2016, 218, 240-245. [CrossRef]

16. Bhatti, M.M.; Zeeshan, A. Study of variable magnetic field and endoscope on peristaltic blood flow of particle-fluid suspension through an annulus. Biomed. Eng. Lett. 2016, 6, 242-249. [CrossRef]

17. Maskeen, M.M.; Mehmood, O.U.; Zeeshan, A. Hydromagnetic solid-liquid pulsatile flow through concentric cylinders in a porous medium. J. Visual. 2018, 21, 407-419. [CrossRef]

18. Ellahi, R.; Zeeshan, A.; Hussain, F.; Abbas, T. Two-phase couette flow of couple stress fluid with temperature dependent viscosity thermally affected by magnetized moving surface. Symmetry 2019, 11, 647. [CrossRef]

19. Zeeshan, A.; Hussain, F.; Ellahi, R.; Vafai, K. A study of gravitational and magnetic effects on coupled stress bi-phase liquid suspended with crystal and Hafnium particles down in steep channel. J. Mol. Liquids 2019, 286, 110898. [CrossRef]

20. Prakash, J.; Tripathi, D.; Tiwari, A.K.; Sait, S.M.; Ellahi, R. Peristaltic pumping of nanofluids through a tapered channel in a porous environment: Applications in blood flow. Symmetry 2019, 11, 868. [CrossRef]

21. Riaz, A.; Bhatti, M.M.; Ellahi, R.; Zeeshan, A.; M Sait, S. Mathematical Analysis on an Asymmetrical Wavy Motion of Blood under the Influence Entropy Generation with Convective Boundary Conditions. Symmetry 2020, 12, 102. [CrossRef]

22. Gireesha, B.; Mahanthesh, B.; Gorla, R.S.R.; Manjunatha, P. Thermal radiation and Hall effects on boundary layer flow past a non-isothermal stretching surface embedded in porous medium with non-uniform heat source/sink and fluid-particle suspension. Heat Mass Transf. 2016, 52, 897-911. [CrossRef]

23. Bhatti, M.; Zeeshan, A.; Ijaz, N.; Bég, O.A.; Kadir, A. Mathematical modelling of nonlinear thermal radiation effects on EMHD peristaltic pumping of viscoelastic dusty fluid through a porous medium duct. Eng. Sci. Technol. Int. J. 2017, 20, 1129-1139. [CrossRef]

24. Kumar, K.G.; Rudraswamy, N.; Gireesha, B.; Manjunatha, S. Non linear thermal radiation effect on Williamson fluid with particle-liquid suspension past a stretching surface. Results Phys. 2017, 7, 3196-3202. [CrossRef]

25. Bhatti, M.; Zeeshan, A.; Ellahi, R.; Shit, G. Mathematical modeling of heat and mass transfer effects on MHD peristaltic propulsion of two-phase flow through a Darcy-Brinkman-Forchheimer porous medium. Adv. Powder Technol. 2018, 29, 1189-1197. [CrossRef]

26. Mahanthesh, B.; Gireesha, B. Scrutinization of thermal radiation, viscous dissipation and Joule heating effects on Marangoni convective two-phase flow of Casson fluid with fluid-particle suspension. Results Phys. 2018, 8, 869-878. [CrossRef]

27. Zhu, L.T.; Liu, Y.X.; Luo, Z.H. An enhanced correlation for gas-particle heat and mass transfer in packed and fluidized bed reactors. Chem. Eng. J. 2019, 374, 531-544. [CrossRef] 
28. Bhatti, M.; Ellahi, R.; Zeeshan, A.; Marin, M.; Ijaz, N. Numerical study of heat transfer and Hall current impact on peristaltic propulsion of particle-fluid suspension with compliant wall properties. Mod. Phys. Lett. B 2019, 33, 1950439. [CrossRef]

29. Horne, A.W.; Critchley, H.O. Mechanisms of disease: The endocrinology of ectopic pregnancy. Exp. Rev. Mol. Med. 2012, 14. [CrossRef]

30. Srivastava, L.; Srivastava, V. Peristaltic transport of blood: Casson model-II. J. Biomech. 1984, 17, 821-829. [CrossRef]

31. Akbar, N.S.; Butt, A.W. Heat transfer analysis of Rabinowitsch fluid flow due to metachronal wave of cilia. Results Phys. 2015, 5, 92-98. [CrossRef]

32. Manzoor, N.; Bég, O.A.; Maqbool, K.; Shaheen, S. Mathematical modelling of ciliary propulsion of an electrically-conducting Johnson-Segalman physiological fluid in a channel with slip. Comput. Methods Biomech. Biomed. Eng. 2019, 685-695. [CrossRef] [PubMed]

33. Chamkha, A.J.; Al-Subaie, M.A. Hydromagnetic buoyancy-induced flow of a particulate suspension through a vertical pipe with heat generation or absorption effects. Turk. J. Eng. Environ. Sci. 2010, 33, 127-134.

34. Marble, F.E. Dynamics of dusty gases. Ann. Rev. Fluid Mech. 1970, 2, 397-446. [CrossRef]

(C) 2020 by the authors. Licensee MDPI, Basel, Switzerland. This article is an open access article distributed under the terms and conditions of the Creative Commons Attribution (CC BY) license (http://creativecommons.org/licenses/by/4.0/). 